Document downloaded from:

http://hdl.handle.net/10251/146880

This paper must be cited as:

Ortigosa, N.; Pérez-Roselló, V.; Donoso, V.; Osca Asensi, J.; Martínez-Dolz, L.; Fernández Rosell, C.; Galbis Verdu, A. (2018). Early prediction of cardiac resynchronization therapy response by non-invasive electrocardiogram markers. Medical \& Biological Engineering \& Computing. 56(4):611-621. https://doi.org/10.1007/s11517-017-1711-1

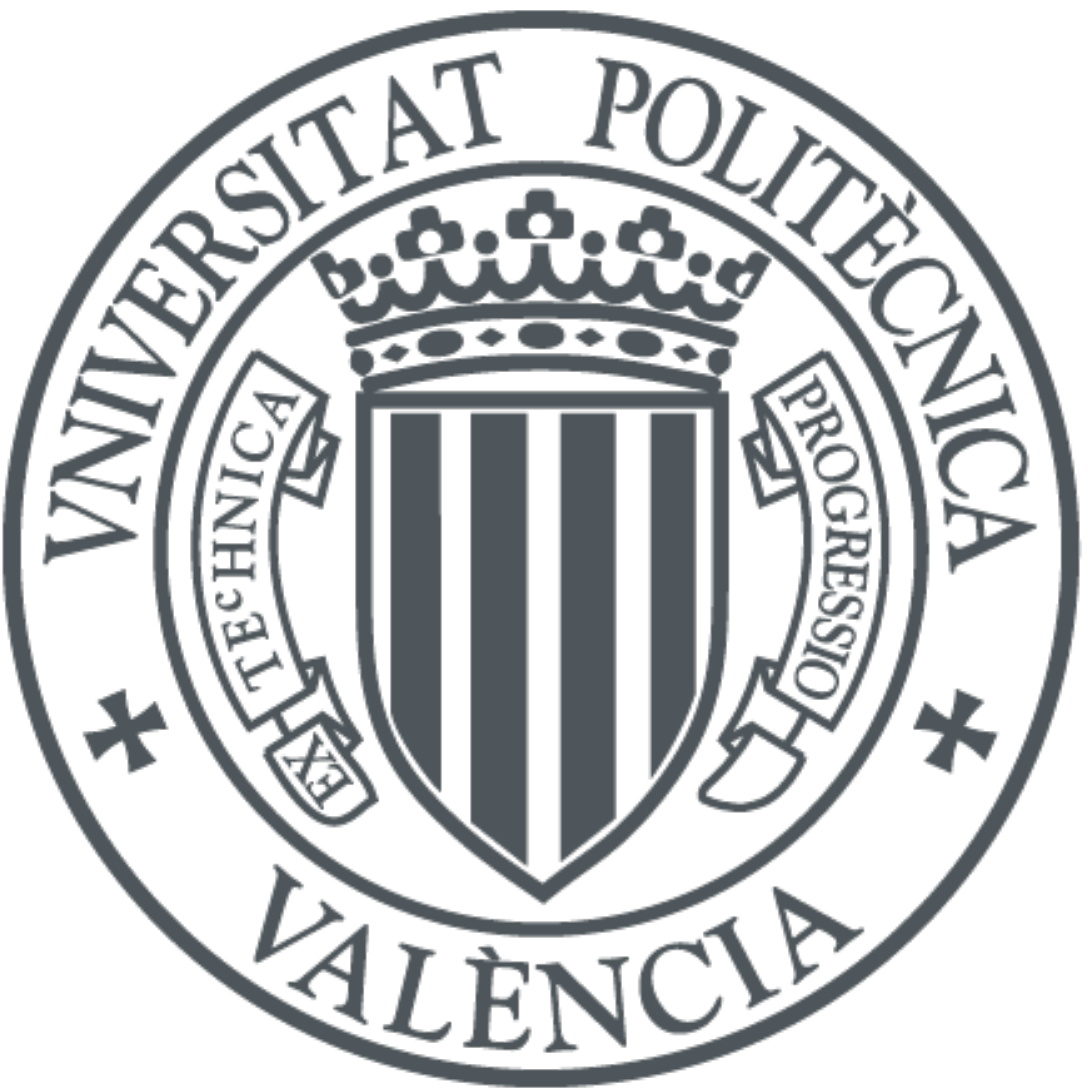

The final publication is available at

https://doi.org/10.1007/s11517-017-1711-1

Copyright Springer-Verlag

Additional Information 


\title{
Early prediction of Cardiac Resynchronization Therapy response by non-invasive electrocardiogram markers
}

\author{
Nuria Ortigosa - Víctor Pérez-Roselló . \\ Víctor Donoso · Joaquín Osca • Luis \\ Martínez-Dolz • Carmen Fernández • \\ Antonio Galbis
}

Received: date / Accepted: date

\begin{abstract}
Cardiac Resynchronization Therapy (CRT) is an effective treatment for those patients with severe heart failure. Regrettably, there are about one third of CRT "non-responders", i.e. patients who have undergone this form of device therapy but do not respond to it, which adversely affects the utility and cost-effectiveness of CRT. In this paper we assess the ability of a novel surface ECG marker to predict CRT response. We performed a retrospective exploratory study of the ECG previous to CRT implantation in 43 consecutive patients with ischemic (17) or non-ischemic (26) cardiomyopathy. We extracted the QRST complexes and obtained a measure of their energy by means of spectral analysis. This ECG marker showed statistically significant lower values for non-responders patients and, joint with the duration of QRS complexes (the current gold-standard to predict CRT response), the following performances: $86 \%$ accuracy, $88 \%$ sensitivity, $80 \%$ specificity. In this manner, the proposed ECG marker may help clinicians to predict positive response to CRT in a non-invasive way, in order to minimize unsuccessful procedures.
\end{abstract}

Keywords Cardiac Resynchronization Therapy · Heart Failure · Electrocardiogram.

This work was supported by MINECO under grants MTM2013-43540-P and MTM201676647-P.

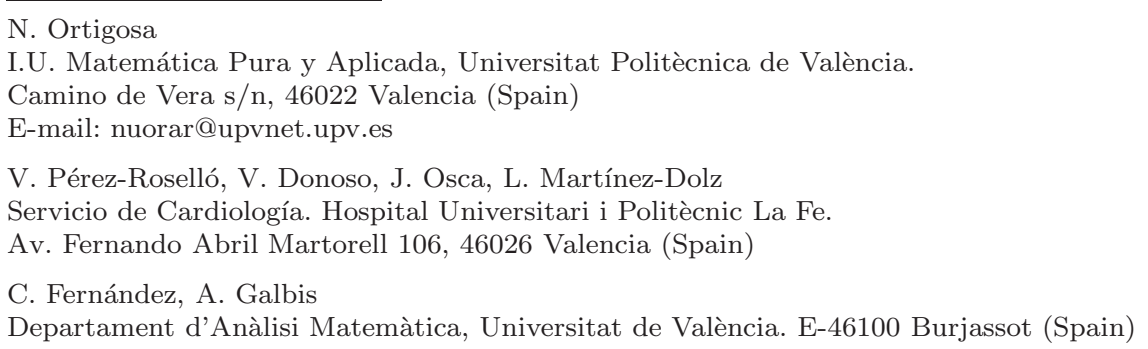




\section{Introduction}

Heart failure (HF) is a clinical syndrome generated by a reduced output and or elevated intracardiac pressures at rest or during stress that results in wellknown signs and symptoms [21]. HF is generally associated with reduced left ventricular ejection fraction (LVEF) [30,18], although a significant proportion of $\mathrm{HF}$ patients presents a preserved left ventricular systolic function [5]. It has been estimated that one third of patients with $\mathrm{HF}$ and left ventricular (LV) systolic dysfunction additionally suffer ventricular dyssynchrony, which deteriorates, even more, the performance of cardiac output. LV dyssynchrony is related to a QRS prolongation, especially in presence of left bundle branch block (LBBB), and worsens the prognosis of HF patients [5].

Cardiac resynchronization therapy (CRT) is a well-accepted therapy for patients with HF, low LVEF and QRS prolongation [8,21,25]. Biventricular pacing is associated with an improved quality of life [9], increased functional capacity [25], reduction in hospitalization for heart failure, and increased survival $[21,8]$. At present, the indication with a higher level of evidence is for symptomatic patients with heart failure in sinus rhythm with a QRS duration $\geq 130 \mathrm{~ms}$, with LBBB, and LVEF $\leq 35 \%$ despite optimal medical therapy [2]. Nevertheless, CRT is also indicated with lesser level of evidence in patients with a non-LBBB pattern in the ECG $[8,31]$.

Despite the impressive effects of CRT, a significant proportion of patients (up to 30-35\%) do not respond to CRT therapy ("non-responders") adversely affecting the utility and cost-effectiveness of this form of device therapy for HF [32]. There are many suggested reasons for an inadequate response to CRT such as persistence of LV dyssynchrony after CRT, phrenic nerve stimulation, lead dislodgement, or suboptimal programming of device $[8,10,27,15]$.

Consequently, there is still a need to improve in the selection of patients to optimize the response to CRT. Prediction of response to CRT has been analysed in many references of the state-of-the-art [33]. Although cardiac imaging is becoming an interesting alternative to ECG analysis to predict CRT response, the surface ECG is still the most studied, since it is a routinely record used in clinical practice.

Recent references have pointed to the area under the QRS complexes of orthogonal vectorcardiograms as good predictors of CRT implantation $[12,29$, $22]$. Other authors prefer to also include the area of the T-wave when defining these new prediction features $[17,28,13]$. Nevertheless, all of them agree in the fact that CRT responders are prone to have larger areas under the curves than CRT non-responders.

In this paper we present a new marker based on energy of the QRS complex and T-wave calculated on the time-frequency spectrum of the surface ECG in order to improve prediction results of response of heart failure patients undergoing CRT implantation.

The rest of the paper is organized as follows. The study population and its clinical characteristics are described in Section 2. The new ECG marker and the classification process are detailed in Sections 3.1-3.3. Next, experimental 
results and performances are depicted in Section 4. Finally, discussion of results is presented in Section 5 and conclusions are drawn in Section 6 .

\section{Materials}

An open-label, single-center, retrospective exploratory study was designed. The study population consisted of 43 consecutive patients (mean age 67 years, 27 men) with heart failure, treated with CRT, as per current guidelines. All patients were symptomatic with most of them in NYHA (New York Heart Association) functional class II or III (93\%). Underlying etiology was ischemic and non-ischemic cardiomyopathy in $40 \%$ and $60 \%$ of patients, respectively.

The prevalence of hypertension was $67 \%$, diabetes $28 \%$, dyslipidaemia $58 \%$. $24 \%$ of patients were current smokers and $43 \%$ obese. The prevalence of atrial fibrillation was $23 \%$ and oral anticoagulants use $19 \%$.

Medication included diuretics in $67 \%$, angiotensin converting enzyme inhibitors in $83 \%$, beta-blockers in $74 \%$, and spironolactone in $48 \%$ of patients.

We defined response to CRT as an increase in LVEF (left ventricular ejection fraction) $\geq 5 \%$ or one who presented an improvement in New York Heart Association (NYHA) functional class $\geq 1$ in absence of death by any cardiovascular cause, heart transplantation or hospital admission for heart failure during a follow-up period of one year. We consider as super-responders those patients with an increase in LVEF $\geq 15 \%$ without cardiovascular death, heart transplantation or hospital admission for heart failure.

According to these criteria 33 out of 43 patients were clinically judged as responders to CRT. 2 patients died and 4 were hospitalized for heart failure. The detailed baseline characteristics of the patients are detailed in table 1.

Surface ECG was acquired in a tertiary center (Hospital Universitari i Politècnic La Fe, Valencia) and bipolar lead II was delineated and analysed. Duration of ECG segments was 5 seconds, which is a common duration for 6 $\times 2$ printout displays [20]. 
Table 1 Distribution of patients included in the retrospective exploratory study, showing mean values or median and interquartile range. LBBB, left bundle branch block; RBBB, right bundle branch block; NIVCD, non-specific intraventricular conduction delay; ACEI, angiotensin-converting enzyme-inhibitor; MRA, mineralocorticoid receptor antagonists. * indicates statistical significance.

\begin{tabular}{|c|c|c|c|c|c|}
\hline & & \multicolumn{2}{|l|}{ Responder } & \multirow[t]{2}{*}{ Total (43) } & \multirow[t]{2}{*}{$\mathrm{p}$-value } \\
\hline & & No $(10)$ & Yes (33) & & \\
\hline Age (years) & & $70(61-77)$ & $66(34-81)$ & $67(34-81)$ & 0.404 \\
\hline \multirow[t]{2}{*}{ Sex } & Male & $7(70.0 \%)$ & $20(60.6 \%)$ & $27(62.8 \%)$ & \multirow{2}{*}{0.719} \\
\hline & Female & $3(30.0 \%)$ & $13(39.4 \%)$ & $16(37.2 \%)$ & \\
\hline \multirow{2}{*}{ Cardiomyopathy } & Ischemic & $7(70.0 \%)$ & $10(30.3 \%)$ & $17(39.5 \%)$ & \multirow{2}{*}{$0.034^{*}$} \\
\hline & Non ischemic & $3(30.0 \%)$ & $23(69.7 \%)$ & $26(60.5 \%)$ & \\
\hline \multirow{4}{*}{ NYHA class } & $\mathrm{I}$ & $0(0.0 \%)$ & $1(3.0 \%)$ & $1(2.3 \%)$ & \multirow{4}{*}{0.349} \\
\hline & II & $4(40.0 \%)$ & $14(42.4 \%)$ & $18(41.9 \%)$ & \\
\hline & III & $4(40.0 \%)$ & $18(54.5 \%)$ & $22(51.2 \%)$ & \\
\hline & IV & $2(20.0 \%)$ & $0(0.0 \%)$ & $2(4.7 \%)$ & \\
\hline \multirow{4}{*}{ ECG pattern } & LBBB & $6(60.0 \%)$ & $26(78.8 \%)$ & $32(74.4 \%)$ & \multirow{4}{*}{$0.005^{*}$} \\
\hline & RBBB & $3(30.0 \%)$ & $0(0.0 \%)$ & $3(7.0 \%)$ & \\
\hline & NICVD & $1(10.0 \%)$ & $1(3.0 \%)$ & $2(4.7 \%)$ & \\
\hline & Stimulated & $0(0.0 \%)$ & $6(18.2 \%)$ & $6(14.0 \%)$ & \\
\hline LBBB & & $6(60.0 \%)$ & $26(78.8 \%)$ & $33(76.7 \%)$ & 0.248 \\
\hline LVEF (average values in \%) & & $24(10-35)$ & $22(10-37)$ & $23(10-37)$ & 0.536 \\
\hline Hypertension & & $9(90.0 \%)$ & $20(60.6 \%)$ & $29(67.4 \%)$ & 0.128 \\
\hline Diabetes & & $6(60.0 \%)$ & $6(18.2 \%)$ & $12(27.9 \%)$ & $0.017^{*}$ \\
\hline Dyslipidaemia & & $8(80.0 \%)$ & $17(51.5 \%)$ & $25(58.1 \%)$ & 0.153 \\
\hline \multirow{3}{*}{ Tobacco use } & Yes (current) & $2(20.0 \%)$ & $8(24.2 \%)$ & $10(23.3 \%)$ & \multirow{3}{*}{0.657} \\
\hline & No & $6(60.0 \%)$ & $17(51.5 \%)$ & $23(53.5 \%)$ & \\
\hline & Ex-smoker & $2(20.0 \%)$ & $8(24.2 \%)$ & $10(23.3 \%)$ & \\
\hline Obesity & & $2(20 \%)$ & $15(45.5 \%)$ & $17(39.5 \%)$ & 0.145 \\
\hline Kidney failure & & $3(30 \%)$ & $5(15.2 \%)$ & $8(18.6 \%)$ & 0.362 \\
\hline Creatinine level $(\mathrm{mg} / \mathrm{dL})$ & & $1.27(0.77-2.11)$ & $1.26(0.59-5.26)$ & $1.25(0.59-5.26)$ & 0.391 \\
\hline Haemoglobin $(\mathrm{mg} / \mathrm{dL})$ & & $11.75(8.8-15.0)$ & $13.67(10-16.5)$ & $13.08(8.8-16.5)$ & $0.009^{*}$ \\
\hline Atrial fibrillation & & $5(50.0 \%)$ & $5(15.2 \%)$ & $10(23.3 \%)$ & $0.036^{*}$ \\
\hline ACEI's use & & $7(70.0 \%)$ & $28(84.8 \%)$ & $35(81.4 \%)$ & 0.094 \\
\hline Beta blockers use & & $5(50.0 \%)$ & $26(78.8 \%)$ & $31(72.1 \%)$ & 0.328 \\
\hline MRA's use & & $3(30.0 \%)$ & $17(51.5 \%)$ & $20(46.5 \%)$ & 0.284 \\
\hline Diuretics use & & $5(50.0 \%)$ & $23(69.7 \%)$ & $28(65.1 \%)$ & 0.259 \\
\hline Oral anticoagulation & & $2(20.0 \%)$ & $6(18.2 \%)$ & $8(18.6 \%)$ & 1.0 \\
\hline
\end{tabular}




\section{Methods}

\subsection{ECG pre-processing}

Baseline and powerline interference were first removed from the surface ECG signals. In particular, baseline wander was reduced by means of cubic splines [19]. Then, we proceed to delineate the ECG in order to detect onset and endset of QRS complexes and T-waves.

\subsection{QRST measure of energy}

Once the delineation is completed, we proceed to calculate the frequency information along the duration of the QRST segments, in order to estimate a measure of their energy. This is done by using a dyadic sampling scheme [3] of the Stockwell Transform (ST) [26], whose analytic expression is:

$$
S(\tau, \nu)=|\nu| \int_{-\infty}^{\infty} g_{0}(\nu(t-\tau)) e^{-2 \pi i \nu t} h(t) d t
$$

where $g_{0}$ denotes a Gaussian window, and $\tau$ and $\nu$ are the indexes for time and frequency, respectively. Stockwell transform is a frequency-adaptive version of the Short-Time Fourier transform, where the window's width varies with frequency. The window is narrower for high frequencies (allowing good time resolution) and its width is enlarged for low frequencies (allowing good frequency resolution). This feature, known as progressive resolution, aims to deal with the uncertainty principle.

The dyadic sampling scheme proposed in [3] allows to have a fast, nonredundant implementation of the ST, called General Fourier-family Transform (GFT). This transform was chosen instead of another approaches with progressive resolution (such as the Discrete Wavelet Transform -DWT-) because ST (and its discrete implementations) not only also have progressive resolution, but are able to provide real frequency information. DWT does not use sinusoidal basis functions, so it measures a kind of scale information, but not frequency information directly. The combination of progressive resolution and globally referenced frequency and phase measurements inherent to ST and its discrete implementations (as GFT) are two desirable properties particularly interesting for some biomedical signal analysis applications, such as the one proposed in this paper.

The ECG marker proposed calculates an energy measure based in the GFT coefficients $(z)$ normalized by the square of the central frequency of each frequency band under analysis $f_{c}^{2}$, according to Proposition 3.4 in [1]. Thus, the proposed marker $E$ is obtained as:

$$
E=\sum_{f=4}^{f=B} \frac{\sum_{t=0}^{t=N-1}|z(t, f)|^{2}}{f_{c}^{2}}
$$


where $N$ is the total number of time windows for each frequency band, and $B$ denotes the number of frequency bands of the time-frequency spectrum. Due to the characteristics of the power spectral density of the ECG, bands under analysis are proposed to start in band number 4 (since this means to study frequencies beyond $2 \mathrm{~Hz}$, where the QRST complexes present their larger power spectral density).

Finally, the proposed feature to predict CRT response is obtained as the median of all markers for the number $n$ of the QRST complexes in the ECG recordings of each patient: Energy measure $=\operatorname{Median}\left\{E_{k}\right\}$

Figure 1 depicts an example of QRST segment from V1 lead and its corresponding time-frequency spectrum, where the different values of the spectral coefficients can be analysed according to a colour map.

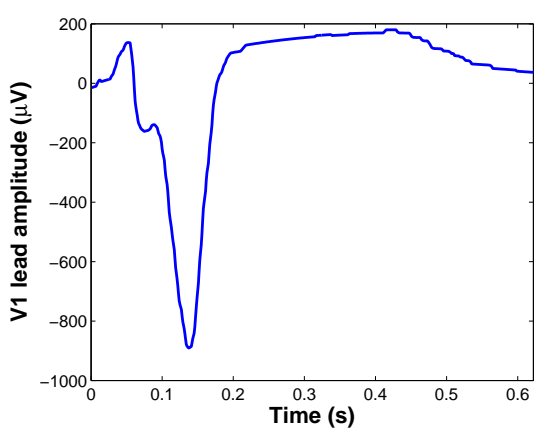

(a)

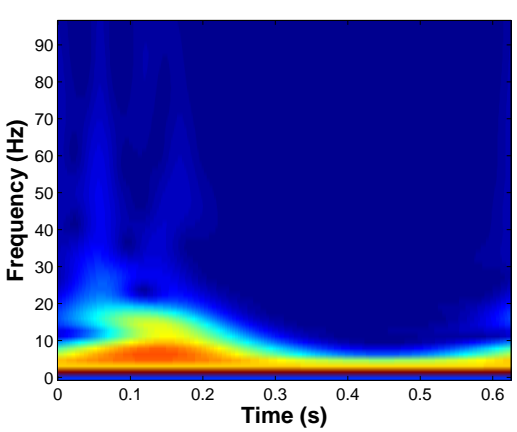

(b)

Fig. 1 (a) Example of QRST extracted from one of the subjects included in the study. (b) Time-frequency spectrum obtained by means of the Stockwell Transform of the QRST segment shown in (a) with cold and warm colours corresponding to small and large ST coefficients, respectively.

\subsection{Classification}

Once the energy measurements of QRST and the duration of QRS complexes have been calculated, they are normalized in order to proceed to classify them using a support vector machine (SVM). We have used the LIBSVM library implemented in $\mathrm{C}++$ and offered by Chang and Lin [6] with a non-linear kernel (radial basis function), since its use minimizes numerical difficulties.

Due to the unbalanced number of patients of each group under analysis (33 responders and 10 non-responders), the direct application of the leavingone-out technique to provide classification results was not suitable. Therefore, we used an adaptive synthetic sampling approach for learning from this type of data sets called AdaSyn [16]. The main difference of AdaSyn and other synthetic oversampling techniques, such as SMOTE [7], is that AdaSyn provides 
not only a balanced representation of the data distribution, but also forces the learning algorithm to focus on the minority data class.

In our case, AdaSyn provides 24 additional synthetic samples for the nonresponders subgroup. Thus, we will apply the leaving-one-out classification over a total data set of 67 samples (33 original responders and 34 non-responders -10 original and 24 synthetic-). In this manner, each sample is classified with the support vector machine resulting from training with the remaining 66 samples. Detailed results over the whole group of samples and only for the original dataset are detailed in Section 4.

\section{Results}

\subsection{Performance measures}

Once the SVM classifier has been trained to maximize global accuracy, several quantitative tests were obtained to assess its performance: sensitivity (rate of responders correctly classified), specificity (rate of non-responders properly classified), false positive rate (FPR), false negative rate (FNR), and F-score (harmonic mean of precision and sensitivity):

$$
\begin{gathered}
\text { Accuracy }=\frac{T P+T N}{T P+T N+F P+F N} \\
\text { Sensitivity }=\frac{T P}{T P+F N} \\
\text { Specificity }=\frac{T N}{T N+F P} \\
F P R=\frac{F P}{F P+T N} \\
F N R=\frac{F N}{F N+T P} \\
F-\text { score }=\frac{2 * T P}{2 * T P+F P+F N}
\end{gathered}
$$

where $T P$ and $T N$ are the responders and non-responders correctly classified, whereas $F P$ and $F N$ denote the non-responders and the responders misclassified.

Classification performance is also depicted by means of the receiver operating characteristic (ROC) curve of the classifier, which illustrates Sensitivity against (1-Specificity) for different thresholds, and the area under the ROC curve. 
4.2 Experimental results

The dataset of this retrospective exploratory study consisted of 43 subjects who suffered from heart failure and who were clinically considered as candidates to be responders to CRT according to the current guidelines [21].

After the delineation of QRST complexes and average the energy measure for each subject, we proceeded to obtain synthetic samples by means of AdaSyn and then perform the classification of the data using a SVM with the leaving-one-out technique. Figure 2 depicts the samples of the real patients and compares them with the synthetic samples obtained using AdaSyn.

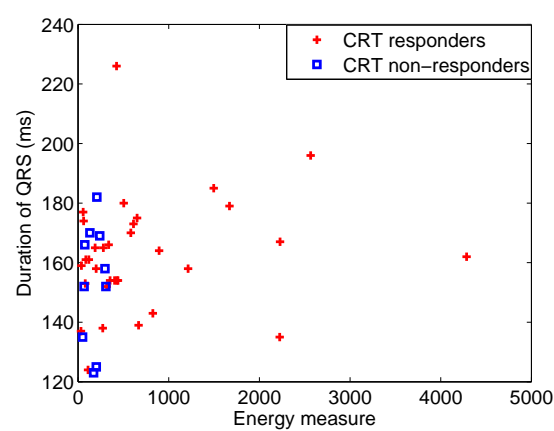

(a)

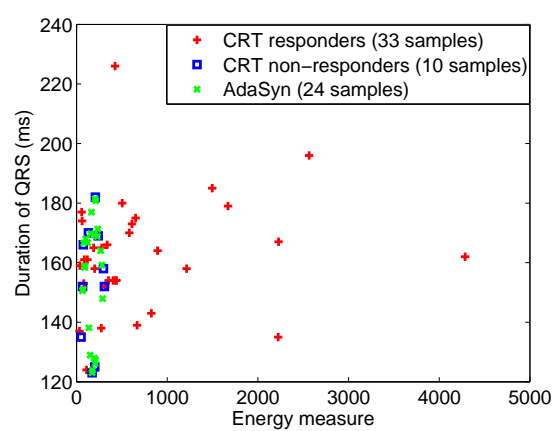

(b)

Fig. 2 (a) Features extracted from the surface ECGs of 43 patients previously to CRT implantation. (b) Features extracted from the surface ECGs of 43 patients previously to CRT implantation and synthetic points generated by AdaSyn [16] in order to balance the number of subjects from each group (CRT responders and CRT non-responders). 


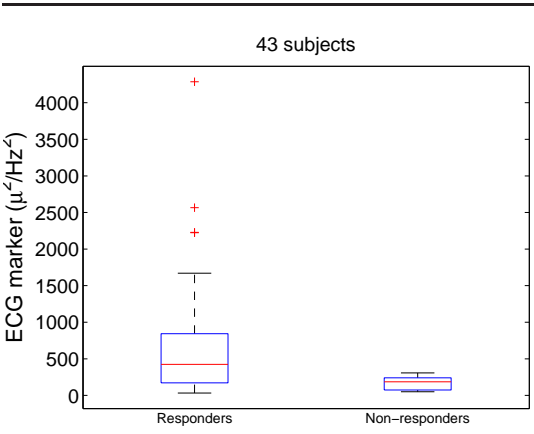

(a)

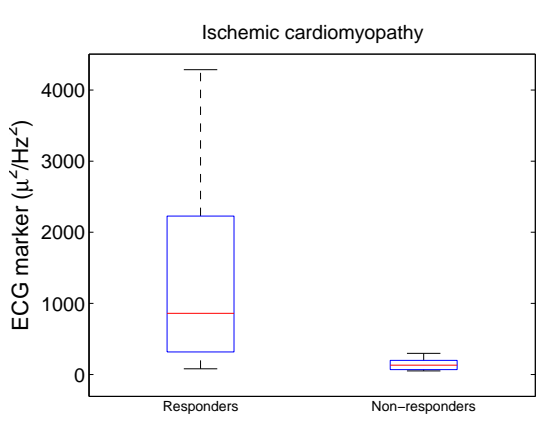

(c)

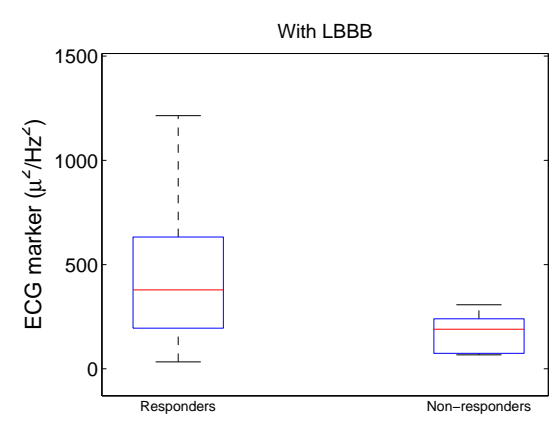

(e)

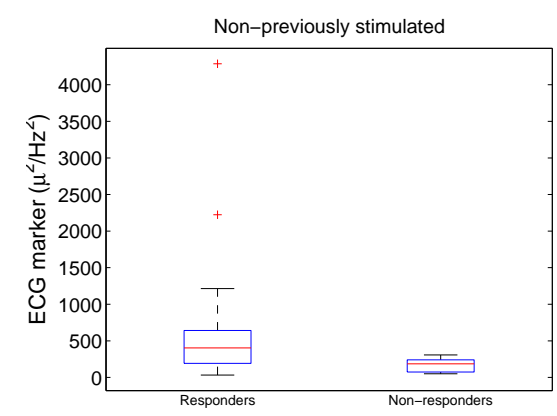

(g)

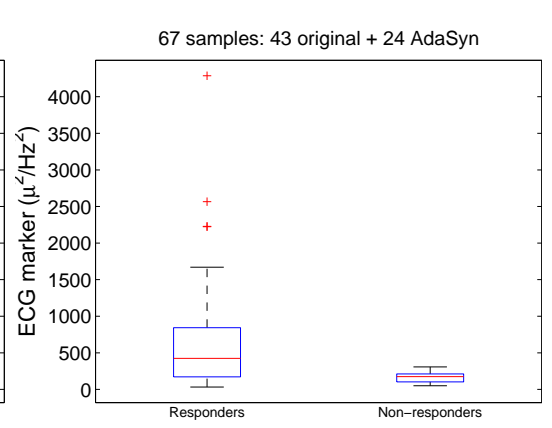

(b)

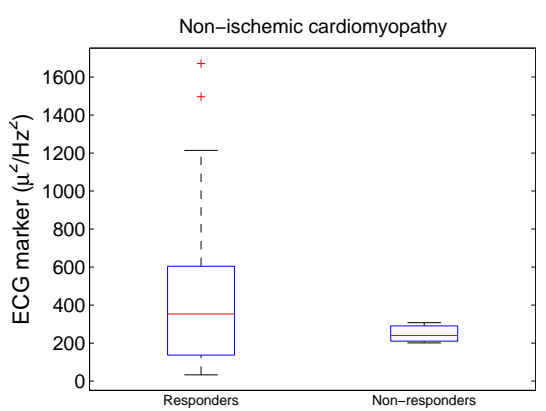

(d)

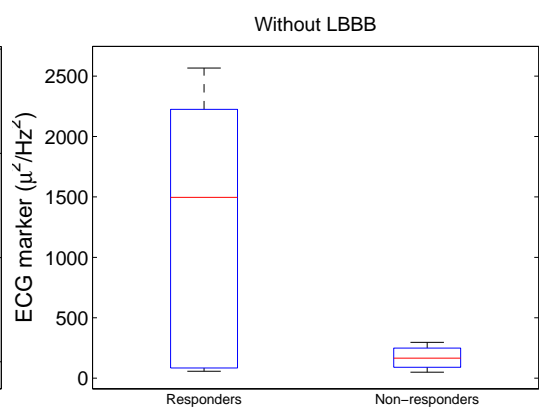

(f)

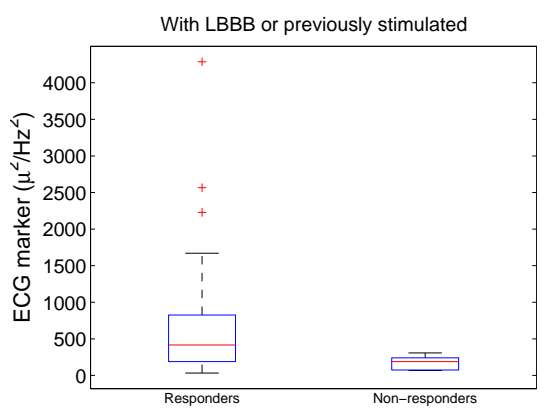

(h)

Fig. 3 Boxplot comparing CRT responders and CRT non-responders energy of QRST complexes. (a) 43 subjects included in the study population. (b) 43 subjects included in the study population and 24 synthetic samples corresponding to CRT non-responders obtained by AdaSyn. (c) Subjects with ischemic cardiomyopathy. (d) Subjects with non-ischemic cardiomyopathy. (e) Subjects with left bundle branch block. (f) Subjects without left bundle branch block. (g) Subjects without pacemaker prior to CRT implantation. (h) Subjects with pacemarker prior to CRT implantarion or with left bundle branch block. 


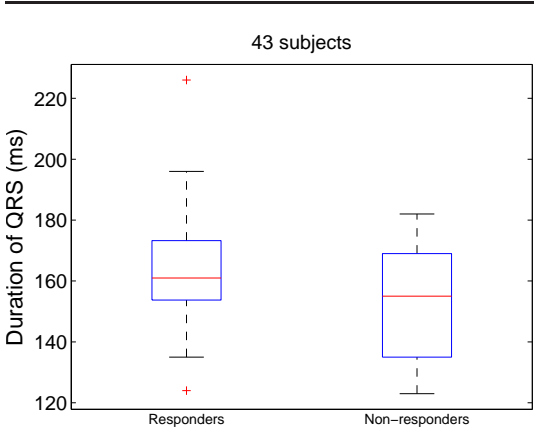

(a)

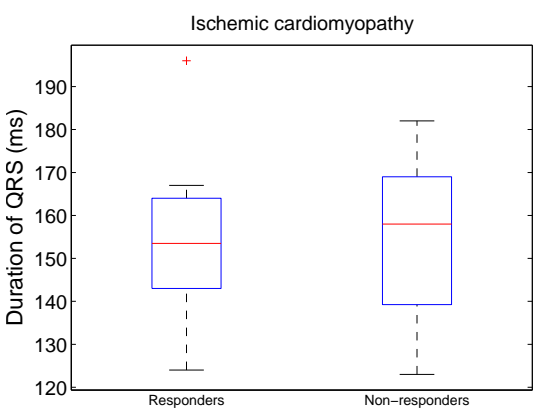

(c)

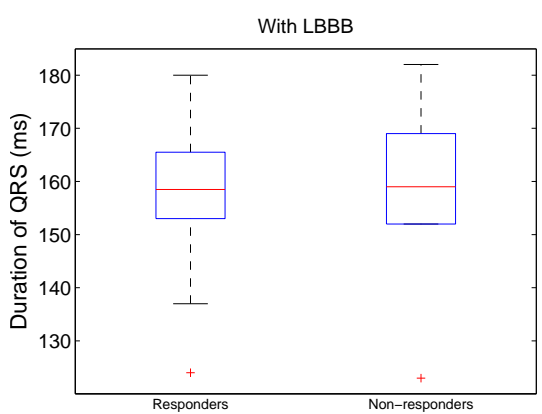

(e)

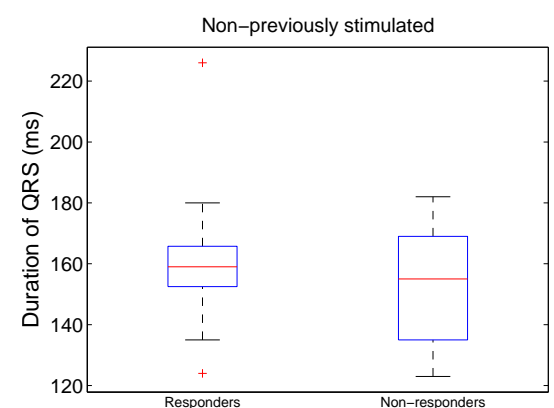

$(\mathrm{g})$

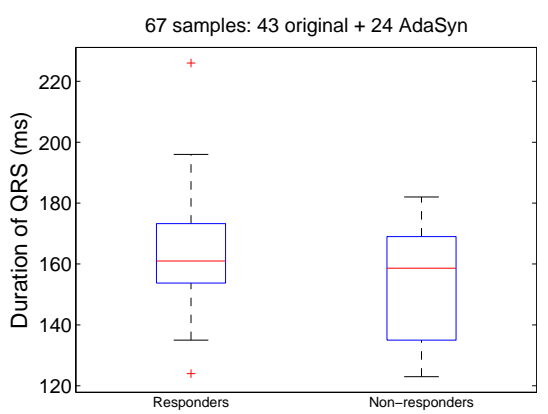

(b)

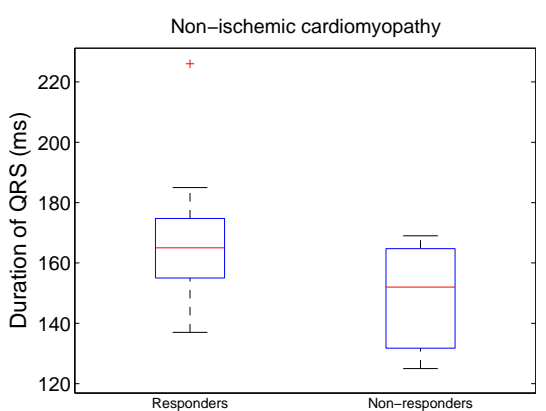

(d)

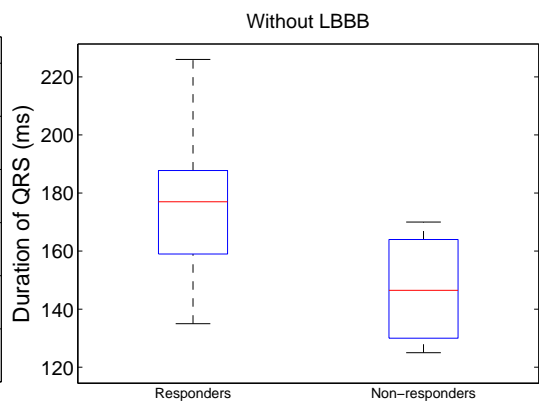

(f)

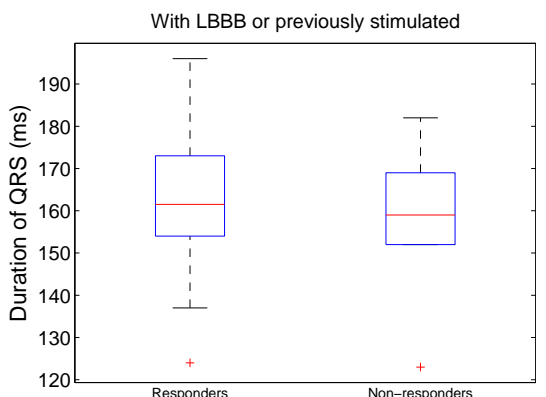

(h)

Fig. 4 Boxplot comparing CRT responders and CRT non-responders duration of QRS complexes. (a) 43 subjects included in the study population. (b) 43 subjects included in the study population and 24 synthetic samples corresponding to CRT non-responders obtained by AdaSyn. (c) Subjects with ischemic cardiomyopathy. (d) Subjects with non-ischemic cardiomyopathy. (e) Subjects with left bundle branch block. (f) Subjects without left bundle branch block. (g) Subjects without pacemaker prior to CRT implantation. (h) Subjects with pacemarker prior to CRT implantarion or with left bundle branch block. 
Table 2 shows the values of the features under analysis for CRT responders and CRT non-responders before CRT implantation, including detailed information for the different subgroups too. These figures indicate that CRT responders are characterized by larger values of the proposed measure of energy of QRST. CRT responders' energy of QRST is about the double of CRT non-responders'. Indeed, it is specially larger for those patients who have ischemic cardiomyopathy.

Wilcoxon statistical test has been calculated revealing that, for the proposed ECG marker based on QRST complexes, p-values are statistically significant (smaller than 0.05) for the general classification (responders vs nonresponders) and for the different subgroups according clinical characteristics, except for patients with dilated cardiomyopathy (Table 2). This larger p-value may be due to the small number of CRT non-responders patients with dilated cardiomyopathy (only 3 subjects), and it will be discussed in Section 5 .

Figures 3 and 4 display the boxplots of the energy measure of QRST complexes and duration of QRS, respectively. Boxplots from Figure 3 show that the proposed energy measure is able to discriminate patients responders to CRT from those non-responders (a-b), specially for subjects with ischemic cardiomyopathy (c), with independence of the presence of LBBB (e-f) or previous implantation of pacemaker $(\mathrm{g}-\mathrm{h})$. These results are in concordance with figures and statistical analysis from Table 2.

Regarding Figure 4, which shows boxplots for the current gold-standard for CRT, it is more difficult to identify the clinical features for which the duration of QRS is able to early distinguish CRT responders from non-responders. Only for the general classification (a), for non-ischemic cardiomyopathy (d), and patients without LBBB (f), boxplots show significant differences in mean. This is also reflected on p-values obtained from statistical analysis shown in Table 2.

The SVM classification results obtained after applying the leaving-one-out technique over the cohort of 43 patients and the AdaSyn synthetic samples are shown in Table 3. Several performances are also included, and results are also detailed for several groups depending on clinical characteristics. Good performances are obtained, presenting an accuracy of $86 \%$, and specificity and sensitivity also above $80 \%$ ( $88 \%$ and $80 \%$, respectively), whereas the rates for false positives and false negatives remain small (about the $15 \%$ in average). Finally, the receiver operating characteristic curve (ROC) and the area under it $(0.8515)$ are shown in Figure 5. The discussion about the obtained results will be enlarged in the subsequent Section. 
Table 2 Features analysed (median measure of energy of QRST complexes and average QRS duration) before CRT implantation for the 43 patients included in the retrospective exploratory study. Statistical significant differences are denoted by ${ }^{*}$, and p-values are obtained for Wilcoxon statistical test. LBBB is left bundle branck block

\begin{tabular}{lccl}
\hline & Responders & Non-responders & p-value \\
\hline ECG marker for QRST & & & \\
\hline All patients & 747.32 & 174.69 & $0.0134^{*}$ \\
Only non previously stimulated & 613.44 & 174.69 & $0.0141^{*}$ \\
Only previously stimulated & 1349.80 & - & - \\
Ischemic cardiomyopathy & 1397.30 & 142.73 & $0.0068^{*}$ \\
Non-ischemic cardiomyopathy & 464.71 & 249.25 & 0.5408 \\
Patients with LBBB & 576.16 & 178.05 & $0.0500^{*}$ \\
Patients without LBBB & 1203.80 & 169.65 & 0.1986 \\
\hline QRS duration (ms) & & & \\
\hline All patients & 162.36 & 153.20 & 0.2742 \\
Only non previously stimulated & 147.85 & 159.30 & 0.5376 \\
Only previously stimulated & 193.93 & - & - \\
Ischemic cardiomyopathy & 155 & 155.14 & 0.8835 \\
Non-ischemic cardiomyopathy & 165.57 & 148.67 & 0.1719 \\
Patients with LBBB & 157.46 & 157.33 & 1 \\
Patients without LBBB & 175.44 & 147 & 0.0892 \\
\hline
\end{tabular}

Table 3 Classification results for the proposed measure of energy of QRST complexes and duration of QRS using the leaving-one-out technique to train the SVM with 67 samples (43 corresponding to the patients and 24 to synthetic samples obtained by AdaSyn [16]). Results are detailed for the 43 patients included in the retrospective exploratoty study, and also including the synthetic samples.

\begin{tabular}{lcccccc}
\hline & Accuracy & Sensitivity & Specificity & FPR & FNR & F-score \\
\hline All 43 patients & 0.8605 & 0.8788 & 0.8000 & 0.2000 & 0.1212 & 0.9063 \\
Non-previously stimulated & 0.8649 & 0.8889 & 0.8000 & 0.2000 & 0.1111 & 0.9057 \\
Previously stimulated & 0.8333 & 0.8333 & - & - & 0.1667 & 0.9091 \\
Ischemic cardiomyopathy & 0.7647 & 0.7000 & 0.8571 & 0.1429 & 0.3000 & 0.7778 \\
Non-ischemic cardiomyopathy & 0.9231 & 0.9565 & 0.6667 & 0.3333 & 0.0435 & 0.9565 \\
With LBBB & 0.8667 & 0.8750 & 0.8333 & 0.1667 & 0.1250 & 0.9130 \\
Without LBBB & 0.8462 & 0.8889 & 0.7500 & 0.2500 & 0.1111 & 0.8889 \\
With LBBB and/or stimulated & 0.8611 & 0.8667 & 0.8333 & 0.1667 & 0.1333 & 0.9123 \\
43patients + AdaSyn samples & 0.8060 & 0.8788 & 0.7353 & 0.2647 & 0.1212 & 0.8169 \\
\hline
\end{tabular}




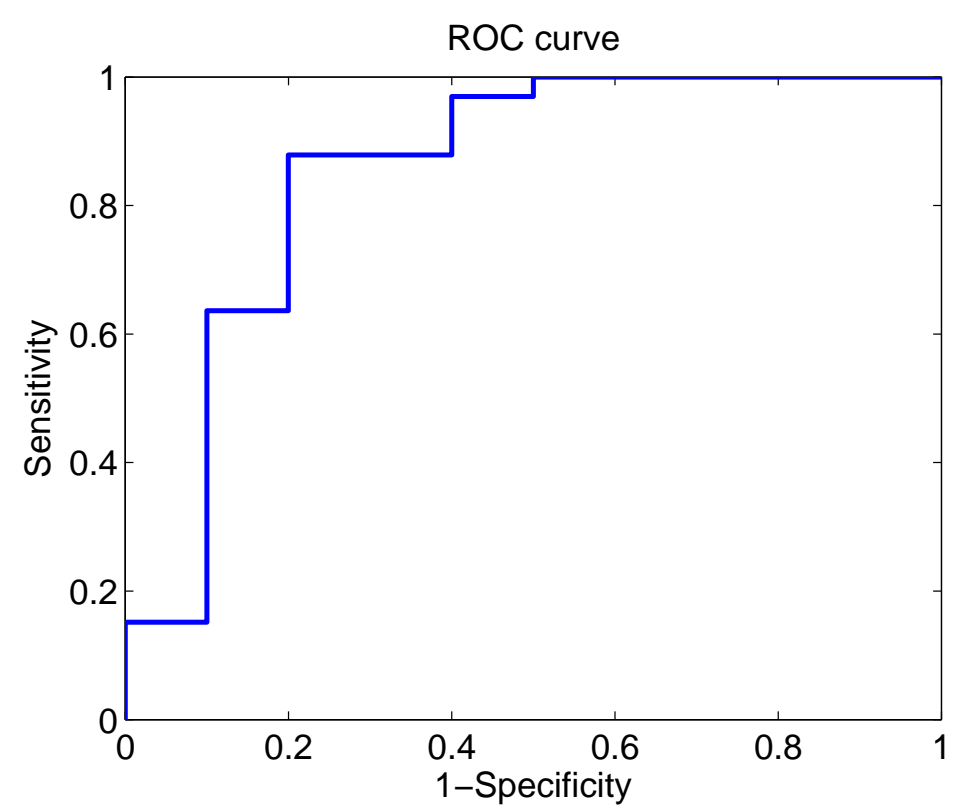

Fig. 5 Receiver operating characteristic curve for the proposed prediction method. Classification has been performed using the leaving-one-out technique. Area under ROC curve is 0.8515 .

\section{Discussion}

In this paper we have presented a new surface ECG marker to predict CRT response in order to improve results of the most accepted prediction marker (the QRS duration). Good performances have been obtained, with an improvement of about $20 \%$ on accuracy and sensitivity with respect to only applying the QRS duration marker, which is the current gold-standard used nowadays in clinical practice.

Clinical guidelines [2] remark the importance of identifying the correct evidences that indicate those patients who are suitable for CRT. Apart from the duration of QRS interval, intracardiac measurements (electrograms) made during the placement of the left ventricle lead [24] and cardiac imaging [4] are able to predict long-term clinical outcome to CRT. Unfortunately, multimodality imaging is still far from daily clinical practice, and electrograms require an invasive proceeding. Thus, in this paper we have proposed a new marker to predict CRT response prior the pacer implantation using the surface ECG, which is a cheap and routinary used record in clinical practice.

Previous references, including $[29,12,22]$ have recently pointed to complement or even replace the ECG markers (such as QRS duration and morphology) by potentially better ECG-based markers to select the optimal candidates to undergo for CRT treatment. These markers are mainly based on the area 
under the QRS curve calculated on the orthogonal vectorcardiogram. Other references point to the area under the QRST, showing prediction odds of response larger for patients with larger area $[28,17]$.

Results presented in this paper are in line with these recent references, since the area under the QRST curve is related with the energy measurement proposed. In this manner, we have shown that responders to CRT also present larger values of energy than non-responders previously to CRT implantation.

In addition, results also confirm their improvement when including the T-wave in the analysis besides QRS complex. Table 4 shows classification results when only studying QRS complexes, showing poorer performances than when T-wave is also considered (Table 3). This can be due to information of repolarization of the ventricles, and further studies will be done regarding it.

Moreover, Table 2 shows significant differences for the subgroup of patients with ischemic cardiomyopathy meanwhile Table 3 presents better results for patients who have non-ischemic cardiomyopathy than for ischemic. This may be due to the small number of patients non-responders to CRT with non-ischemic cardiomyopathy who were included in the cohort under study. Patients with ischemic cardiomyopathy are a common population in trials evaluating CRT and current guidelines also recommend CRT in ischemic patients. On the other side, it has been published that ischemic cardiomyopathy is associated to a lower probability to respond to CRT [23], so the results that we have observed are in accordance to previous findings.

Regarding other clinical characteristics, patients with RBBB represent about 20 to $30 \%$ of patients involved in trials evaluating CRT, and current guidelines also recommend CRT with a recommendation IIa in RBBB patients when QRS is wide enough. On the other hand, it has been published that RBBB patients are associated to a lower probability to respond to CRT [11], so the results that we can observe in Table 1 (30\% of non-responders having RBBB) are in accordance to previous findings.

Present guidelines also recommend CRT with a recommendation IIa in atrial fibrillation patients when the rest of patient characteristics are present. Nevertheless, recent references have published that AF patients are associated to a lower probability of response to $\mathrm{CRT}$. So, the prevalence of AF in the study population ( $50 \%$ in non-responders while it was $15.2 \%$ in the responders group, detailed in Table 1) is in accordance with previous findings.

As above-mentioned, the most interesting result that we have observed is that in a population with a clear indication for CRT, the new ECG marker has the ability to provide an early identification of patients responders and nonresponders to CRT. On the other hand, the selection of the proposed single parameter may also integrate the information contained in the classic duration of QRS, which could represent an advantage, either in well-studied indications of CRT (wide QRS with LBBB) or even especially in cases where the classic gold-standard does not correspond to an indication with more evidence (for example, patients with non-specific intraventricular conduction delay, or less prolonged durations of QRS). Future work will include thorough studies on this, since non-specific intraventricular conduction delay pathophysiology 
(which ischemic cardiomyopathy is most often associated with) is less studied than LBBB, and for which results of CRT implantation are contradictory [14].

Table 4 Classification results for the proposed energy of measure of QRS complexes and their duration using the leaving-one-out technique to train the SVM with 67 samples (43 corresponding to the patients and 24 to synthetic samples obtained by AdaSyn [16]). Results are detailed for the 43 patients included in the retrospective exploratory study, and also including the synthetic samples.

\begin{tabular}{lcccccc}
\hline & Accuracy & Sensitivity & Specificity & FPR & FNR & F-score \\
\hline All 43 patients & 0.7674 & 0.7576 & 0.8000 & 0.2000 & 0.2424 & 0.8333 \\
Non-previously stimulated & 0.7297 & 0.7037 & 0.8000 & 0.2000 & 0.2963 & 0.7917 \\
Previously stimulated & 1 & 1 & - & - & 0 & 1 \\
Ischemic cardiomyopathy & 0.7059 & 0.7000 & 0.7143 & 0.2857 & 0.3000 & 0.7368 \\
Non-ischemic cardiomyopathy & 0.8077 & 0.7826 & 1 & 0 & 0.2174 & 0.8780 \\
With LBBB & 0.7333 & 0.7500 & 0.6667 & 0.3333 & 0.2500 & 0.8182 \\
Without LBBB & 0.8462 & 0.7778 & 1 & 0 & 0.2222 & 0.8750 \\
With LBBB and/or stimulated & 0.7778 & 0.8000 & 0.6667 & 0.3333 & 0.2000 & 0.8571 \\
43patients + AdaSyn samples & 0.7015 & 0.7576 & 0.6471 & 0.3529 & 0.2424 & 0.7143 \\
\hline
\end{tabular}

\section{Conclusions}

In this paper we have presented a retrospective exploratory study of patients with severe heart failure who have undergone cardiac resynchronization therapy. Nowadays, the ECG is still the main complementary exploration used to select the patients who can benefit from CRT. Indeed, it is not less important the identification of patients with higher probability of non-response to CRT, since more advanced therapies (such as ventricular assist devices) can be used as alternatives when conventional medical treatment and CRT are not sufficient.

Therefore, the correct selection of the patients is key not only to allow an early identification of non-responders (which would avoid unnecessary delays in the use of more advanced therapies), but also to maximize the costeffectiveness of CRT.

We have analysed the ability of a new marker based on energy of QRS complexes and $\mathrm{T}$ waves to predict the positive response to CRT before biventricular pacemaker implantation. It has been shown that this marker is significantly larger for CRT responders than for non-responders to CRT, obtaining early classification accuracy of $86 \%$. Thus, our exploratory study presents an additional parameter to the classic morphology and duration of QRS, which allows the evaluation of the potential response that a certain patient may have to CRT in an objective and reproducible way. 
Further studies with larger populations are needed, but presented results may open a door to reduce the percentage of non-successful CRT implantations (nowadays about $30 \%$ ) with using a non-invasive technique as the surface ECG.

\section{References}

1. P. Boggiatto, C. Fernández, and A. Galbis. A Group Representation Related to the Stockwell Transform. Indiana University Mathematics Journal, 58(5):2277-2296, 2009.

2. M. Brignole, A. Auricchio, G. Baron-Esquivias, P. Bordachar, and G. Boriani et al. 2013 ESC Guidelines on cardiac pacing and cardiac resynchronization therapy. Europace, 15:1070-1118, 2013.

3. R.A. Brown, M.L. Lauzon, and R. Frayne. A General Description of Linear TimeFrequency Transforms and Formulation of a Fast, Invertible Transform That Samples the Continuous S-Transform Spectrum Nonredundantly. IEEE Trans. Signal Process., 58(1):281-290, January 2010.

4. P. Carità, E. Corrado, G. Pontone, A. Curnis, and L. Bontempi et al. Non-responders to cardiac resynchronization therapy: Insights from multimodality imaging and electrocardiography. A brief review. Int J Cardiol., 225:402-407, 2016.

5. S. Cazeau, C. Leclercq, T. Lavergne, S. Walker, C. Varma, and C. Linde et al. Effects of multisite biventricular pacing in patients with heart failure and intraventricular conduction delay. N Engl J Med, 344:873-880, 2001.

6. C.C. Chang and C.J. Lin. LIBSVM: A library for support vector machines. ACM Transactions on Intelligent Systems and Technology, 2(3):27:1-27:27, 2011.

7. N.V. Chawla, K.W. Bowyer, L.O. Hall, and W.P.Kegelmeyer. SMOTE: Synthetic Minority Over-Sampling Technique. Journal of Artificial Intelligence Research, 16(1):321357, 2002.

8. J.G.F. Cleland, W.T. Abraham, C. Linde, M.R. Gold, and J.B Young et al. An individual patient meta-analysis of five randomized trials assessing the effects of cardiac resynchronization therapy on morbidity and mortality in patients with symptomatic heart failure. Eur. Heart Journal, 34(46):3547-3556, December 2013.

9. J.G.F. Cleland, M.J. Calvert, Y. Verboven, and N. Freemantle. Effects of cardiac resynchronization therapy on long-term quality of life: an analysis from the Cardiac Resynchronisation-Heart Failure (CARE-HF) study. Am Heart J, 157:457-466, 2009.

10. J.G.F. Cleland, N. Freemantle, E. Erdmann, D. Gras, L. Kappenberger, and et al. Long-term mortality with cardiac resynchronization therapy in the Cardiac Resynchronization-Heart Failure (CARE-HF) trial. Eur J Heart Fail, 14:628-634, 2012.

11. C.A. Egoavil, R.T. Ho, A.J. Greenspon, and B.B. Pavri. Cardiac resynchronization therapy in patients with right bundle branch block: Analysis of pooled data from the MIRACLE and Contak CD trials. Heart Rhythm, 2(6):611-615, June 2005.

12. E.B. Engels, M. Mafi-Rad, A.M. van Stipdonk, K. Vernooy, and F.W. Prinzen. Why QRS Duration Should Be Replaced by Better Measures of Electrical Activation to Improve Patient Selection for Cardiac Resynchronization Therapy. J Cardiovasc Transl Res., 9(4):257-265, 2016.

13. E.B. Engels, E.M. Végh, C.J. Van Deursen, K. Vernooy, J.P. Singh, and F.W. Prinzen. T-wave area predicts response to cardiac resynchronization therapy in patients with left bundle branch block. J Cardiovasc Electrophysiol., 26(2):176-183, 2015.

14. R. Eschalier, S. Ploux, P. Ritter, M. Haïssaguerre, K.A Ellenbogen, and P. Bordachar. Nonspecific intraventricular conduction delay: Definitions, prognosis, and implications for cardiac resynchronization therapy. Heart Rhythm, 12(5):1071-1079, 2015.

15. I. Goldenberg, V. Kutyifa, H.U. Klein, D.S. Cannom, M.W. Brown, and et al. Survival with cardiac-resynchronization therapy in mild heart failure. N Engl J Med, 370:16941701, 2014.

16. H. He, Y. Bai, E.A. Garcia, and S. Li. ADASYN: Adaptive Synthetic Sampling Approach for Imbalanced Learning. pages 1322-1328. Int'l. J. Conf. Neural Networks, 2008. 
17. J. Jacobsson, R. Borgguist, C. Reitan, E. Ghafoori, and N.A. Chatterjee et al. Usefulness of the Sum Absolute QRST Integral to Predict Outcomes in Patients Receiving Cardiac Resynchronization Therapy. J Cardiovasc Electrophysiol., 118(3):389-395, 2016.

18. J.J. McMurray. Clinical practice. Systolic heart failure. N Engl J Med, 3623:228-238, 2010 .

19. C. R. Meyer and H. N. Keiser. Electrocardiogram baseline noise estimation and removal using cubic splines and state-space computation techniques. Comput. Biomed. Res., 10:459-470, 1977.

20. N. Ortigosa and V.M. Giménez. Raw data extraction from electrocardiograms with portable document format. Comput. Meth. Programs Biomed., 113(1):284-289, January 2014.

21. P. Ponikowski, A.A. Voors, S.D Anker, H. Bueno, J.G. Cleland, and A.J. Coats et al. 2016 ESC Guidelines for the diagnosis and treatment of acute and chronic heart failure: The Task Force for the diagnosis and treatment of acute and chronic heart failure of the European Society of Cardiology (ESC). Developed with the special contribution of the Heart Failure Association (HFA) of the ESC. Eur. J. Heart Fail., 18(8):891-975, August 2016.

22. M.M. Rad, G.W. Wijntjens, E.B. Engels, Y. Blaauw, and J.G. Luermans et al. Vectorcardiographic QRS area identifies delayed left ventricular lateral wall activation determined by electroanatomic mapping in candidates for cardiac resynchronization therapy. Heart Rhythm., 13(1):217-225, 2016.

23. M. Shanks, V. Delgado, and J.J. Bax. Cardiac Resynchronization Therapy in NonIschemic Cardiomyopathy. Journal of Atrial Fibrillation, 8(5):47-52, February 2016.

24. J.P. Singh, D. Fan, E.K. Heist, C.R. Alabiad, and C. Taub et al. Left ventricular lead electrical delay predicts response to cardiac resynchronization therapy. Heart Rhythm, 3(11):1285-1292, 2006.

25. S.M. Sohaib, J.A. Finegold, S.S. Nijjer, R. Hossain, C. Linde, and et al. Opportunity to increase life span in narrow QRS cardiac resynchronization therapy recipients by deactivating ventricular pacing: evidence from randomized controlled trials. JACC Heart Fail, 3:327-336, 2015.

26. R.G. Stockwell, L. Mansinha, and R.P.Lowe. Localization of the complex spectrum: The S transform. IEEE Trans. Signal Process., 44(4):998-1001, April 1996.

27. A.S.L. Tang, G.A. Wells, M. Talajic, M.O. Arnold, R. Sheldon, and et al. Cardiacresynchronization therapy for mild-to-moderate heart failure. $N$ Engl $\mathrm{J} \mathrm{Med,} \mathrm{363:2385-}$ 2395, 2010.

28. L.G. Tereshchenko, A. Cheng, J. Park, N. Wold, T.E. Meyer, and M.R. Gold et al. Novel measure of electrical dyssynchrony predicts response in cardiac resynchronization therapy: Results from the SMART-AV Trial. Heart Rhythm, 12(2):2402-2410, 2015.

29. C.J. van Deursen, K. Vernooy, E. Dudink, L. Bergfeldt, and H.J. Crijns et al. Vectorcardiographic QRS area as a novel predictor of response to cardiac resynchronization therapy. J Electrocardiol., 48(1):45-52, 2015.

30. T.J. Wang. Natural history of asymptomatic left ventricular systolic dysfunction in the community. Circulation, 108:977-982, 2003.

31. B. Woods, N. Hawkins, S. Mealing, A. Sutton, W.T. Abraham, and et al. Individual patient data network meta- analysis of mortality effects of implantable cardiac devices. Heart, 101:1800-1806, 2015.

32. C. Ypenburg, R.J. van Bommel, C.J. Borleffs, G.B. Bleeker, and E.Boersma et al. Longterm prognosis after cardiac resynchronization therapy is related to the extent of left ventricular reverse remodeling at midterm follow-up. J Am Coll Cardiol, 53(6):483-490, February 2009.

33. C.M. Yu and D.L. Hayes. Cardiac resynchronization therapy: state of the art 2013. European Heart Journal, 34:1396-1403, 2013. 
Nuria Ortigosa received the MSc in Telecommunications Engineering in 2007 and a $\mathrm{PhD}$ in 2011, both from Universitat Politècnica de València. Her current research focuses on time-frequency analysis and its applications to biomedical signal processing.

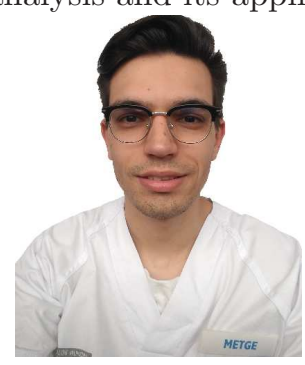

Víctor Pérez-Roselló received the degree in Medicine and Surgery in 2014 from Universitat Autònoma de Barcelona. Nowadays, he is in his third year of specialization in cardiology (resident doctor) in Hospital Universitari i Politècnic La Fe, Valencia.

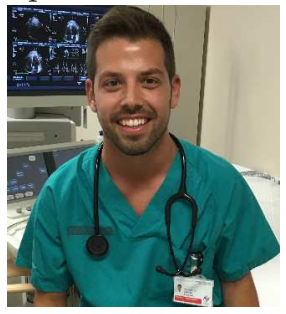

Víctor Donoso received the degree in Medicine and Surgery in 2014 from Universitat Rovira i Virgili de Tarragona (Spain). He is currengtly doing his third year of specialization in cardiology (resident doctor) in Hospital Universitari i Politènic la Fe of Valencia (Spain).

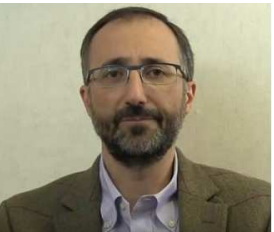

Joaquin Osca received the MSc in Medicine and Surgery in 1995 and a PhD in 2005, both from Universitat de València. His current research focuses on atrial fibrillation and cardiac resynchronization therapy. 


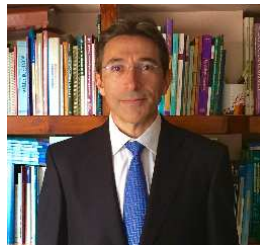

Luis Martínez-Dolz is the head of Cardiology Service of Hospital Universitari i Politècnic La Fe of Valencia (Spain). He is author of more than 200 scientific papers in peer-review journals and associate professor in Faculty of Medicine at Universitat de València.

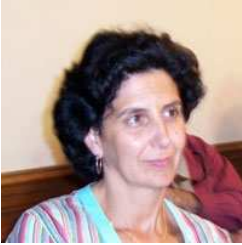

Carmen Fernández is Associate Professor at University of Valencia. Her main research interests are in the field of functional analysis and time-frequency analysis. She is also interested in the study of ECG signals.

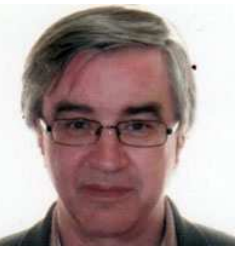

Antonio Galbis is Full Professor and director of the Mathematical Analysis Department at University of Valencia. His research interests include time-frequency analysis, Fourier analysis and applications to biomedical signal processing. 